DESY 98-206

December 1998

\title{
Testing non-perturbative strong interaction effects via the Adler function
}

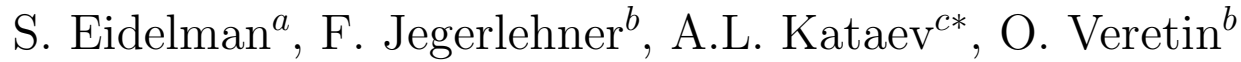 \\ a Budker Institute for Nuclear Physics \\ 630090 Novosibirsk, Russia \\ $b$ Deutsches Elektronen-Synchrotron DESY \\ Platanenallee 6, D-15738 Zeuthen, Germany \\ c Institute for Nuclear Research of the Academy of Sciences of Russia \\ 117312 Moscow, Russia
}

\begin{abstract}
Based on the compilation of the available $e^{+} e^{-}$data [1], we present a non-perturbative estimation of the Adler function derived from the electromagnetic current correlator, and compare it with theoretical predictions from perturbative QCD (pQCD). The comparison is presented for the Euclidean region where pQCD is supposed to work best. We emphasize that such a comparison only makes sense if one takes into account the exact mass dependence of the perturbative predictions, which are available for the leading and next to leading (two-loop) order. In order to have the correct physical mass dependence in the evolution of the strong coupling as well, we utilize the MOM scheme $\beta$-function to two-loops calculated recently [2]. Three-loop effects, which are available as series expansions for low [3] and high [4] momentum transfer, are included by using Padé improvement. We discuss possible constraints on non-perturbative effects as suggested by the operator product expansion.
\end{abstract}

*Partially supported by Landau-Heisenberg program and Russian Foundation of Basic Research, Grants N 96-01-01860, N 96-02-18897 


\section{Introduction}

There are different ways of comparing QCD theoretical predictions with available time-like experimental data for the $e^{+} e^{-}$total cross-section. Among the proposed methods are the global duality sum rules with different smearing functions [5, 6, 7, 8] as well as the local duality sum rules [9, 10]. The Borel sum rules have already been used before to analyze the low-energy $e^{+} e^{-}$data [11] and gave the reasonable value of the gluon condensate $\left\langle G^{2}\right\rangle$ and a rather low value of the parameter $\Lambda_{\frac{(3)}{M S}}$, which turns out to be in contradiction with the current world average value of $\alpha_{s}\left(M_{Z}\right)=0.119 \pm 0.002$ [12].

In view of the improved understanding of the influence of the perturbative QCD corrections (both massless [13, 14] and massive ones [3, 14, 15]) and the appearance of the compilation of the available $e^{+} e^{-}$data [1] it is worthwhile to study the problem of the comparison of the experimental data with the theoretical predictions at the new level.

In this our work we will follow the idea originally proposed in Ref. [16 and implemented in part in Ref. 17 and will use for the comparison with theory the experimental data for the Adler function, defined through the following dispersive relation

$$
D\left(Q^{2}\right)=Q^{2} \int_{4 m_{\pi}^{2}}^{\infty} \frac{R^{\exp }(s)}{\left(s+Q^{2}\right)^{2}} d s,
$$

where $Q^{2}=-q^{2}$ is the squared Euclidean momentum transfer, $s$ is the center of mass energy squared for hadron production in $e^{+} e^{-}$-annihilation,

$$
R(s)=\frac{\sigma_{\text {tot }}\left(e^{+} e^{-} \rightarrow \gamma^{*} \rightarrow \text { hadrons }\right)}{\sigma\left(e^{+} e^{-} \rightarrow \gamma^{*} \rightarrow \mu^{+} \mu^{-}\right)}
$$

the hadronic to leptonic cross section ratio with $\sigma\left(e^{+} e^{-} \rightarrow \gamma^{*} \rightarrow \mu^{+} \mu^{-}\right)=\frac{4 \pi \alpha^{2}}{3 s}, \alpha=e^{2} / 4 \pi$ being the QED fine structure constant.

The attractive features of this procedure were advocated from different points of view in Ref. 18 and Ref.[19].

\section{Calculations of the Adler function}

In this note we reconsider the question of the reliability of pQCD predictions at lower energies (down to about $1 \mathrm{GeV}$ ) by calculating the Adler function associated with the hadronic part of the electromagnetic current

$$
J_{\mu}^{\gamma}=\sum_{q} Q_{q} J_{\mu V}^{q}=\sum_{i}\left(\frac{2}{3} \bar{u}_{i} \gamma_{\mu} u_{i}-\frac{1}{3} \bar{d}_{i} \gamma_{\mu} d_{i}\right) .
$$

By $u_{i}$ and $d_{i}$ we denote the upper and lower components of the quark weak-isodoublets, respectively ( $i$ is the family index).

$$
J_{\mu V}^{q}=\bar{q} \gamma_{\mu} q
$$

is the conserved flavor diagonal vector current of the quark $q$ with charge $Q_{q}$ and mass $m_{q}$. We first define the photon vacuum polarization amplitude $\Pi_{\gamma}^{\prime}\left(q^{2}\right)$ by

$$
\begin{aligned}
\Pi_{\mu \nu}^{\gamma}(q) & =i \int d^{4} x e^{i q x}<0\left|T J_{\mu}^{\gamma}(x) J_{\nu}^{\gamma}(0)\right| 0> \\
& =-\left(q^{2} g_{\mu \nu}-q_{\mu} q_{\nu}\right) \Pi_{\gamma}^{\prime}\left(q^{2}\right) .
\end{aligned}
$$


By $\Pi_{V}^{\prime}$ we denote the corresponding amplitude for the vector current $J_{\mu V}^{q}$.

The Adler function is defined as the derivative

$$
D(-s)=-\left(12 \pi^{2}\right) s \frac{d \Pi_{\gamma}^{\prime}(s)}{d s} .
$$

We may write

$$
D(-s)=\frac{3 \pi}{\alpha} s \frac{d}{d s} \Delta \alpha_{\text {had }}(s)
$$

where

$$
\Delta \alpha_{\text {had }}\left(q^{2}\right)=-\frac{\alpha q^{2}}{3 \pi} \operatorname{Re} \int_{4 m_{\pi}^{2}}^{\infty} d s \frac{R(s)}{s\left(s-q^{2}-i \varepsilon\right)}
$$

is the hadronic contribution to the shift of the fine structure constant at scale $q^{2}$. A careful estimate of this function using the existing experimental data was given some time ago in Ref. [1]. Experimental data for $R(s)$ may be used up to $E_{\text {cut }}=40 \mathrm{GeV}$, for higher energies $\gamma Z$ mixing would complicate the analysis. The high energy tail is calculated by using pQCD. Up to three-loops results are available for massive quarks [20]. The four-loop corrections are known in the approximation of massless quarks [14].

In the present note we are going to evaluate the Adler function by the same procedure. It is important that we work in the Euclidean region $Q^{2}=-q^{2}>0$ where pQCD is supposed to work best.

Before presenting the non-perturbative result, which follows from the estimate of the dispersion integral, we briefly discuss the analytic results which were obtained from perturbative QCD and the operator product expansion (OPE). We write

$$
D\left(Q^{2}\right)=D^{(0)}\left(Q^{2}\right)+D^{(1)}\left(Q^{2}\right)+D^{(2)}\left(Q^{2}\right)+\cdots+D^{\mathrm{NP}}\left(Q^{2}\right)
$$

where $D^{(n)}\left(Q^{2}\right)$ is the $n$-th order pQCD contribution proportional to $\left(\alpha_{s} / \pi\right)^{n}$ and $D^{\mathrm{NP}}\left(Q^{2}\right)$ is the non-perturbative part which will be specified below.

We first consider the pQCD contributions. For the representation of the explicit expressions we use the variables

$$
y=4 m_{f}^{2} / s \quad, \quad \xi=\frac{\sqrt{1-y}-1}{\sqrt{1-y}+1}
$$

where $\xi$ is taking values $0 \leq \xi \leq 1$ for $s \leq 0$.

The $\overline{\mathrm{MS}}$ subtracted transverse photon vacuum polarization function $\Pi_{\gamma}^{\prime}\left(q^{2}\right)$, which defines the full photon propagator as

$$
-g_{\mu \nu} \frac{i}{q^{2}} \frac{1}{1+e^{2} \Pi_{\gamma}^{\prime}\left(q^{2}\right)}
$$

at the one-loop level is given by

$$
e^{2} \Pi_{\gamma}^{\prime}\left(q^{2}\right)=\frac{\alpha}{3 \pi} \sum_{f} Q_{f}^{2} N_{c f}\left(\ln \frac{\mu^{2}}{m_{f}^{2}}+G\right)
$$


with $\mu$ the $\overline{\mathrm{MS}}$ scale parameter, $N_{c f}$ the color factor and

$$
G=\frac{5}{3}+y+\left(1+\frac{y}{2}\right) \sqrt{1-y} \ln \xi
$$

The corresponding one-loop contribution to $\Delta \alpha$ is

$$
\Delta \alpha\left(q^{2}\right)=e^{2}\left(\Pi_{\gamma}^{\prime}(0)-\Pi_{\gamma}^{\prime}\left(q^{2}\right)\right)
$$

while for the one-loop Adler function we obtain

$$
D^{(0)}\left(Q^{2}\right)=\sum_{f} Q_{f}^{2} N_{c f} H^{(0)}
$$

with

$$
H \equiv\left(12 \pi^{2}\right) \dot{\Pi}_{V}^{\prime}, \quad \dot{\Pi}_{V}^{\prime} \equiv-s d \Pi_{V}^{\prime} / d s
$$

in terms of the vector current amplitude $\Pi_{V}^{\prime}$. Explicitly we have

$$
H^{(0)}=y \frac{d G}{d y}=1+\frac{3 y}{2}-\frac{3 y^{2}}{4} \frac{1}{\sqrt{1-y}} \ln \xi .
$$

Asymptotically, we find

$$
H^{(0)} \rightarrow \begin{cases}\frac{1}{5} \frac{Q^{2}}{m_{f}^{2}}-\frac{3}{70}\left(\frac{Q^{2}}{m_{f}^{2}}\right)^{2}+\frac{1}{105}\left(\frac{Q^{2}}{m_{f}^{2}}\right)^{3}+\cdots & Q^{2} \ll m_{f}^{2} \\ 1-6 \frac{m_{f}^{2}}{Q^{2}}-12\left(\frac{m_{f}^{2}}{Q^{2}}\right)^{2} \ln \frac{m_{f}^{2}}{Q^{2}}+24\left(\frac{m_{f}^{2}}{Q^{2}}\right)^{3}\left(\ln \frac{m_{f}^{2}}{Q^{2}}+1\right)+\cdots & Q^{2} \gg m_{f}^{2}\end{cases}
$$

and this behavior determines the quark parton model (QPM) (leading order QCD) property of the Adler function: heavy quarks $\left(m_{f}^{2} \gg Q^{2}\right)$ decouple like $Q^{2} / m_{f}^{2}$ while light modes $\left(m_{f}^{2} \ll Q^{2}\right)$ contribute $Q_{f}^{2} N_{c f}$ to $D^{(0)}$. Using the two-loop QCD vacuum polarization functions given in the appendix we obtain for the two-loop contribution to the Adler function

$$
D^{(1)}\left(Q^{2}\right)=\frac{\alpha_{s}\left(Q^{2}\right)}{\pi} \sum_{f} Q_{f}^{2} N_{c f} H^{(1)}
$$

where $H^{(1)}=\left(12 \pi^{2}\right) \dot{\Pi}_{V}^{\prime}(2)\left(-Q^{2}, m_{f}^{2}\right)$.

The full massive three-loop vector current correlator $\Pi^{\prime(3)}$ is not yet known, however, a sufficient number of terms were calculated both for the small mass expansion [4] as well as for the heavy mass expansion [3]. By taking the derivative (41) we obtain the contribution to the Adler function

$$
D^{(2)}\left(Q^{2}\right)=\left(\frac{\alpha_{s}\left(Q^{2}\right)}{\pi}\right)^{2} \sum_{f} Q_{f}^{2} N_{c f} H^{(2)}
$$

where $H^{(2)}=\left(12 \pi^{2}\right) \dot{\Pi}_{V}^{\prime(3)}\left(-Q^{2}, m_{f}^{2}\right)$.

The convergence of the series expansions break down at Euclidean $Q^{2}=4 m^{2}$, right in the region where mass effects are most important, and we use a conformal mapping together with 
Padé improvement [21] in order to obtain results useful for the present analysis. For details we refer to a brief discussion in the appendix.

A way to parametrize non-perturbative (NP) effects away from resonances, as applicable here, is prescribed by the OPE of the current correlator (3), valid for large enough $Q^{2}$. This part is due to non-vanishing gluon and light quark condensates [7]. They yield the leading power corrections

$$
\begin{aligned}
D^{\mathrm{NP}}\left(Q^{2}\right)= & \sum_{q=u, d, s} Q_{q}^{2} N_{c q}\left(8 \pi^{2}\right) \\
& \cdot\left[\frac{1}{12}\left(1-\frac{11}{18} \frac{\alpha_{s}\left(\mu^{2}\right)}{\pi}\right) \frac{<\frac{\alpha_{s}}{\pi} G G>}{Q^{4}}\right. \\
+ & 2\left(1+\frac{\alpha_{s}\left(\mu^{2}\right)}{3 \pi}+\left(\frac{47}{8}-\frac{3}{4} l_{q \mu}\right)\left(\frac{\alpha_{s}\left(\mu^{2}\right)}{\pi}\right)^{2}\right) \frac{<m_{q} \bar{q} q>}{Q^{4}} \\
+ & \left.\left(\frac{4}{27} \frac{\alpha_{s}\left(\mu^{2}\right)}{\pi}+\left(\frac{4}{3} \zeta_{3}-\frac{88}{243}-\frac{1}{3} l_{q \mu}\right)\left(\frac{\alpha_{s}\left(\mu^{2}\right)}{\pi}\right)^{2}\right) \sum_{q^{\prime}=u, d, s} \frac{<m_{q^{\prime}} \bar{q}^{\prime} q^{\prime}>}{Q^{4}}+\cdots\right]
\end{aligned}
$$

where $l_{q \mu} \equiv \ln \left(Q^{2} / \mu^{2}\right) .<\frac{\alpha_{s}}{\pi} G G>$ and $<m_{q} \bar{q} q>$ are the scale-invariantly defined condensates. The terms beyond leading order in $\alpha_{s}$ have been calculated from the results which were obtained in Refs. [22], [23], [24] and [25]. The terms beyond the leading order in $\alpha_{s}$ are unimportant because experimental data only yield rough constraints on the condensates and we include them for completeness only. Sum rule estimates of the condensates yield [7, 26]

$<\frac{\alpha_{s}}{\pi} G G>\simeq(0.336 \cdots 0.442 \mathrm{GeV})^{4}$ and $<m_{q} \bar{q} q>\simeq\left\{\begin{array}{l}-(0.086 \cdots 0.111 \mathrm{GeV})^{4} \text { for } q=u, d \\ -(0.192 \cdots 0.245 \mathrm{GeV})^{4} \text { for } q=s .\end{array}\right.$

with rather large uncertainties. The values assumed for the quark condensates are based on the recent estimate[27] $<\bar{q} q>\simeq-(235 \pm 27 \mathrm{MeV})^{3}$ and the quark masses $m_{u} \simeq m_{u} \simeq$ $\left(m_{u}+m_{d}\right) / 2 \simeq 7.2 \pm 1.1 \mathrm{MeV}$ and $m_{s} \simeq 175 \pm 25 \mathrm{MeV}$ [28], all at scale $1 \mathrm{GeV}$. Eq. (13) will be evaluated numerically at $\mu^{2}=Q^{2}$.

\section{Numerical results and conclusions}

For numerical studies we used the following pole quark masses [12]

$$
m_{u} \sim m_{d} \sim m_{s} \sim 0 ; m_{c}=1.55 \mathrm{GeV} ; m_{b}=4.70 \mathrm{GeV} ; m_{t}=173.80 \mathrm{GeV} .
$$

For the strong interaction coupling we take $\alpha_{s \overline{\mathrm{MS}}}^{(5)}=0.120 \pm 0.003$ at the scale $M_{Z}=91.19$ $\mathrm{GeV}$ [29]. Up to two-loop we use the exact mass dependence (see e.g. [30]) of the amplitudes as well as of the exact mass dependent two-loop $\bar{\alpha}_{s}\left(Q^{2}\right)$ in the gauge invariant background-field MOM renormalization scheme, presented recently in Ref. [2]. In the transition from the $\overline{\mathrm{MS}}$ to the MOM scheme we adapt the rescaling procedure described in [2], such that for large $\mu$

$$
\bar{\alpha}_{s}\left(\left(x_{0} \mu\right)^{2}\right)=\alpha_{s}\left(\mu^{2}\right)+0+O\left(\alpha_{s}^{3}\right) .
$$

This means that $x_{0}$ is chosen such that the couplings coincide to leading and next-to-leading order at asymptotically large scales. Numerically we find $x_{0} \simeq 2.0144$. Due to this normalization by rescaling the coefficients of the Adler-function remain the same in both schemes up to three-loops. 
The numerical evaluation of the dispersion integral (1) is based on the compilation of data
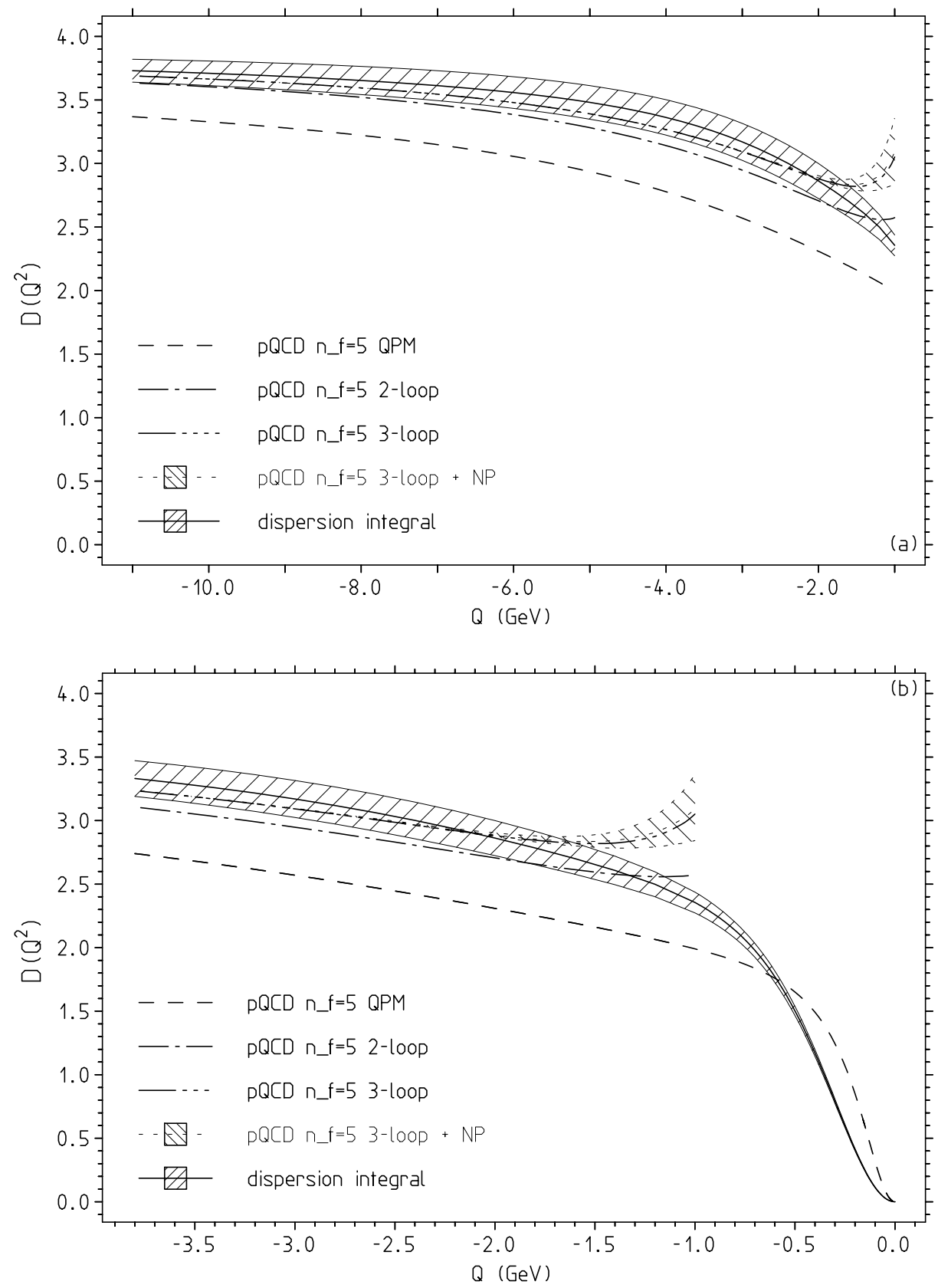

Figures 1a and 1b: We show the "experimental" Adler function together with pQCD predictions and power corrections (NP) in the region below about $10 \mathrm{GeV}$ (a) and separately for the low energy tail (b). The negative sign chosen for $Q$ should remind the reader that we are considering space-like momentum transfer. The shaded area represents the $\pm 1 \sigma$ band obtained from the data. A similar error band is shown for the uncertainties in the power corrections.

discussed in [四. Here we will not use all the data up to $40 \mathrm{GeV}$, however, still take a conservative attitude, and replace data by pQCD results only where it is obviously safe to do so. Accordingly, in the regions from $4.5 \mathrm{GeV}$ to $M_{\Upsilon}$ and above $12 \mathrm{GeV}$ we use pQCD, including the massive results available up to three-loops [国] and the four-loop contribution in the massless 
approximation [14. The "experimental" behavior of the $D$-function is displayed in Figs. 1(a) and $1(\mathrm{~b})$, together with the theoretical predictions. The data and the error analysis utilized in the evaluation of (四) are described in Ref. [回]. Both statistical and systematic errors of the data were taken into account. Note that the meaning of the $1 \sigma$ error band differs from the usual "one standard deviation" since its points at different $Q$ are highly correlated. The origin of the uncertainty is shown in Table 1.

Table 1: Origin of uncertainties for the "experimental" Adler function at $Q=2.5 \mathrm{GeV}$ and $M_{Z}$.

\begin{tabular}{|c||rrr||rrr|}
\hline & $D(2.5 \mathrm{GeV})$ & rel. err. & abs. err. & $D\left(M_{Z}\right)$ & rel. err. & abs. err. \\
\hline Resonances: & $.688(.025)$ & $3.6 \%$ & $0.8 \%$ & $.004(.000)$ & $5.2 \%$ & $0.0 \%$ \\
$E<M_{J / \psi}$ & $1.068(.127)$ & $11.9 \%$ & $4.2 \%$ & $.002(.000)$ & $14.9 \%$ & $0.0 \%$ \\
$M_{J / \psi}<E<3.6 \mathrm{GeV}$ & $.178(.035)$ & $19.9 \%$ & $1.2 \%$ & $.001(.000)$ & $19.8 \%$ & $0.0 \%$ \\
$3.6 \mathrm{GeV}<E<M_{\Upsilon}$ & $.850(.055)$ & $6.4 \%$ & $1.8 \%$ & $.032(.002)$ & $7.0 \%$ & $0.1 \%$ \\
$M_{\Upsilon}<E<12 \mathrm{GeV}$ & $.088(.008)$ & $8.7 \%$ & $0.3 \%$ & $.024(.002)$ & $9.0 \%$ & $0.1 \%$ \\
$E<12 \mathrm{GeV}$ data & $2.871(.146)$ & $5.1 \%$ & $4.8 \%$ & $.063(.003)$ & $4.9 \%$ & $0.1 \%$ \\
$12 \mathrm{GeV}<E \mathrm{QCD}$ & $.162(.001)$ & $0.3 \%$ & $0.0 \%$ & $3.755(.006)$ & $0.2 \%$ & $0.2 \%$ \\
total & $3.033(.146)$ & $4.8 \%$ & $4.8 \%$ & $3.818(.007)$ & $0.2 \%$ & $0.2 \%$ \\
\hline
\end{tabular}

At $M_{Z} D\left(Q^{2}\right)$ is completely dominated be the perturbative high energy tail, the uncertainty is the one of pQCD. As $Q^{2}$ decreases the dominating region moves to lower energies. At $2.5 \mathrm{GeV}$ contributions to $D\left(Q^{2}\right)$ are spread over all energies below $M_{\Upsilon}$ and uncertainties from the region below $M_{J / \psi}$ are dominating. Errors at $2.5 \mathrm{GeV}$ are about a factor 20 larger and dominated by systematic errors of the $e^{+} e^{-}$data.

The agreement between pQCD and the "data" looks rather good, but only after inclusion of the 3-loop contribution. If we had not include the 3-loop term it would appear that there is some room for the non-perturbative power corrections (13). After adding the 3-loop result there is no region left where the power corrections, with the above given values for the parameters lead to an improvement. So, the comparison of the perturbative predictions with the "data" looks quite impressive after inclusion of the 3-loop massive contribution. The inclusion of the top $\left(n_{f}=6\right)$ does not alter the results at the energies shown. We can not see a region of intermediate $Q^{2}$ where adding the non-perturbative power corrections (NP) does improve the agreement of 3-loop pQCD predictions with the presented "data". It also does not look possible to estimate in a convincing way the leading coefficient $\left\langle\frac{\alpha_{s}}{\pi} G G>\right.$. Due to the power behavior, these kind of corrections drop very fast at larger energies, above about $2 \mathrm{GeV}$, while they grow very fast at lower energies, below about $0.75 \mathrm{GeV}$, which signals the breakdown of the applicability of the expansion. In fact, including the $1 / Q^{6}$ terms would lead to a large contribution of opposite sign at low $Q^{2}$. The main problem is that these corrections become substantial only at energies where the perturbation expansion can no longer be trusted.

It should be noted that in the Euclidean region there are no "threshold steps" as they are suggested if the $\overline{\mathrm{MS}}$ scheme is applied. In the $\overline{\mathrm{MS}}$ scheme effective theories with different number of light flavors must be matched at the different scales set by the quark masses [31] in order to restore decoupling of heavy flavors. The physical behavior is illustrated in Fig. 2. 


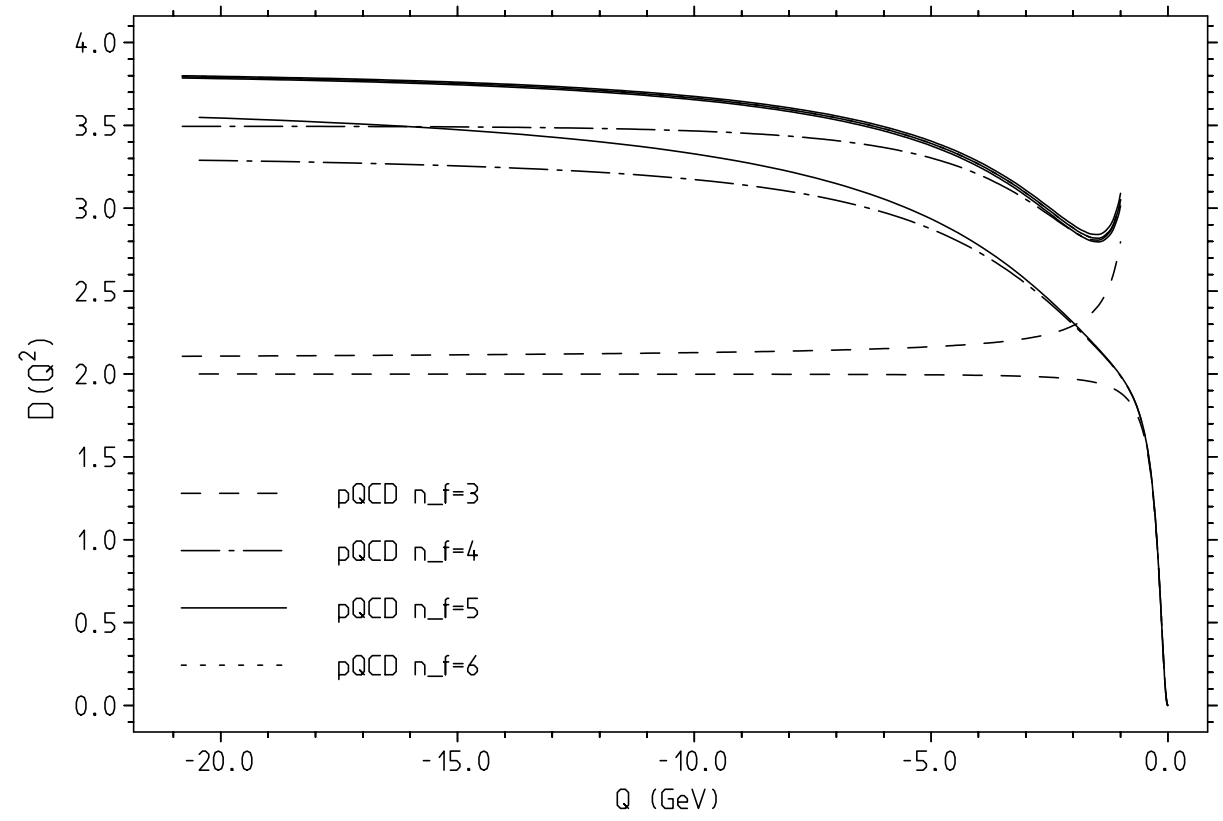

Figure 2: The flavor dependence of the Adler function in pQCD. For each $n_{f}=3 \cdots 6$ two curves are shown: one for the QPM (lower of the two) and the other for pQCD at three-loops (upper of the two). The cases $n_{f}=5$ and $n_{f}=6$ are graphically indistinguishable at the energies shown. The thickness of the $n_{f}=5 O\left(\alpha_{s}^{2}\right)$ "line" illustrates the small theoretical uncertainty obtained from the variation $\alpha_{s}^{(5)} \overline{\mathrm{MS}}=0.120 \pm 0.003$ at the scale $M_{Z}=91.19 \mathrm{GeV}$.

In particular at low momenta the $\overline{\mathrm{MS}}$ prescription does not provide a good description of the physical behavior and hence its applicability in the Euclidean region is worth a critical inspection. In this spirit we also would expect an improvement of the QCD description of deep inelastic scattering in cases where different effective numbers of flavors $n_{f}$ have to be considered [32].

Note that the 3-loop term is quite large and higher orders still could give sizable contributions. The massless 4-loop contribution may be estimated as follows: in massless pQCD we have the familiar result [14] $R(s)=3 \sum_{f} Q_{f}^{2} \times\left(1+a+c_{1} a^{2}+c_{2} a^{3}+\cdots\right)$ with $a=\alpha_{s}(s) / \pi, c_{1}=$ $1.9857-0.1153 n_{f}$ and $c_{2}=-6.6368-1.2002 n_{f}-0.0052 n_{f}^{2}-1.2395\left(\sum Q_{f}\right)^{2} /\left(3 \sum Q_{f}^{2}\right)$. In this approximation $R(s)$ is a constant if we discard the running of $\alpha_{s}$ and we can evaluate (11) obtaining $D\left(Q^{2}\right) \sim R\left(Q^{2}\right)$ at large $Q^{2}$. Numerically the 4-loop term proportional to $c_{2}$ amounts to $-0.06 \%$ at $100 \mathrm{GeV}$ and increases to about $-0.5 \%$ at $2.5 \mathrm{GeV}$. At the low energy this estimate is of course not reliable because we do not expect the approximation to work. Nevertheless in may indicate the possible size of the missing higher order corrections. Due to the increase of the running strong coupling the higher order predictions become unreliable at lower energies. Once we observe that the functional dependence deviates from that of the data, which happens below about $2.5 \mathrm{GeV}$, pQCD is not reliable any longer. The large uncertainties in the data (see Table 1) on the one hand and the beginning failure of pQCD on the other hand, unfortunately do not allow to draw any conclusion about non-perturbative physics. Calculations of missing higher order terms including the mass effects certainly would help a lot to draw more reliable conclusions about the borderline of validity of pQCD. 
Acknowledgments One of us (A.L.K) is grateful to D. V. Shirkov for his interest in the present work and for useful discussions. He (A.L.K) wishes to thank DESY Zeuthen where part of this work was done for its hospitality.

\section{Appendix}

Here we present the explicit formulae for the two-loop vacuum polarization amplitudes of the flavor diagonal vector current. We first introduce the abbreviations

$$
f=-\frac{1}{2} \ln \xi, \quad g=\ln (1-\xi), \quad h=\ln (1+\xi)
$$

where $y$ and $\xi$ are given by (8) and

$$
\Delta \mathrm{Li}_{3}=2 \mathrm{Li}_{3}(\xi)-\mathrm{Li}_{3}\left(\xi^{2}\right), \quad \Delta \mathrm{Li}_{2}=\mathrm{Li}_{2}(\xi)-\mathrm{Li}_{2}\left(\xi^{2}\right)
$$

and define the auxiliary functions

$$
\begin{aligned}
& \mathrm{XX}=\Delta \mathrm{Li}_{3}+\frac{8}{3} f \Delta \mathrm{Li}_{2}+\frac{4}{3} f^{2}(2 h+g), \\
& \mathrm{YY}=\frac{8}{3}\left(\Delta \mathrm{Li}_{2}+2 f(2 h+g)+3 f^{2}\right) .
\end{aligned}
$$

The poly-logarithms $\operatorname{Li}_{n}(z)$ are defined by the integrals

$$
\operatorname{Li}_{n}(z)=\frac{(-1)^{n-1}}{(n-2) !} \int_{0}^{1} \frac{\ln ^{n-2}(x) \ln (1-x z)}{x} d x
$$

and $\zeta_{n}=\zeta(n)=\sum_{i=1}^{\infty} 1 / n^{i}$ is the Riemann zeta-function, with values $\zeta_{2}=\pi^{2} / 6, \zeta_{3}=$ $1.202057 \ldots, \zeta_{4}=\pi^{4} / 90$ etc..

In terms of these functions we find the following compact expression for the two-loop contribution to the Adler function related to the QCD vector neutral current vacuum polarization amplitude: ? $^{2}$

$$
\begin{aligned}
12 \pi^{2} \dot{\Pi}_{V}^{\prime}(2)= & -2 y^{2} \mathrm{XX}+\sqrt{1-y}((1+y) \mathrm{YY}-14 / 3 y f) \\
& +\left(3 y^{2}-4-4 /(1-y)\right) f^{2} \\
& +1+11 / 3 y+2 y^{2} \zeta(3)
\end{aligned}
$$

The following exercise about handling asymptotic expansions is intended to illustrate at the two-loop level the procedure to be applied at the three-loop level where an exact massive result is not yet known.

\footnotetext{
2 it is given by

$$
\dot{\Pi}_{V}^{\prime(2)}=\frac{1}{3}\left(\operatorname{Re} \Pi_{V}^{N C}(s) / s-\operatorname{Re} \dot{\Pi}_{V}^{N C}(s)\right)
$$
}

in terms of the amplitudes which we can find in Sec. 4.5 of Ref. [30] (see also [33]). There the rules for the analytic continuation to the time-like region can also be found. 
The expansion for large $Q^{2}\left(y=-4 m^{2} / Q^{2} \rightarrow 0\right)$ reads $\left(\mathrm{L} \equiv \ln \left(Q^{2} / m^{2}\right)\right)$

$$
\begin{aligned}
12 \pi^{2} \dot{\Pi}_{V}^{\prime(2)} & =1+3(1-\mathrm{L}) y+\left(\frac{17}{24}+2 \zeta(3)+\frac{1}{4} \mathrm{~L}-\frac{3}{2} \mathrm{~L}^{2}\right) y^{2} \\
& +\left(\frac{139}{108}+\frac{19}{9} \mathrm{~L}-\frac{29}{24} \mathrm{~L}^{2}\right) y^{3}+\left(\frac{2173}{13824}+\frac{2575}{1152} \mathrm{~L}-\frac{203}{192} \mathrm{~L}^{2}\right) y^{4} \\
& +\left(-\frac{8311}{25600}+\frac{75727}{34560} \mathrm{~L}-\frac{1169}{1152} \mathrm{~L}^{2}\right) y^{5}+\left(-\frac{784577}{1382400}+\frac{100063}{46080} \mathrm{~L}-\frac{383}{384} \mathrm{~L}^{2}\right) y^{6} \\
& +\cdots,
\end{aligned}
$$

and for low $Q^{2}$ we have

$$
\begin{aligned}
12 \pi^{2} \dot{\Pi}_{V}^{(2)} & =-\left(\frac{328}{81} y^{-1}+\frac{3592}{675} y^{-2}+\frac{999664}{165375} y^{-3}+\frac{831776}{127575} y^{-4}+\frac{1729540864}{252130725} y^{-5}\right. \\
& \left.+\frac{43321977728}{6087156075} y^{-6}+\frac{401009026048}{54784404675} y^{-7}+\frac{5064168384512}{676610809875} y^{-8}+\cdots\right)
\end{aligned}
$$

Both series expansions diverge at the boundary of the circle of convergence $Q^{2}=4 \mathrm{~m}^{2}$. In fact this is precisely in the region where mass effects are of the order of unity in the Euclidean region. We therefore apply a conformal mapping

$$
y^{-1}=\frac{-Q^{2}}{4 m^{2}} \rightarrow \omega=\frac{1-\sqrt{1-1 / y}}{1+\sqrt{1-1 / y}}
$$

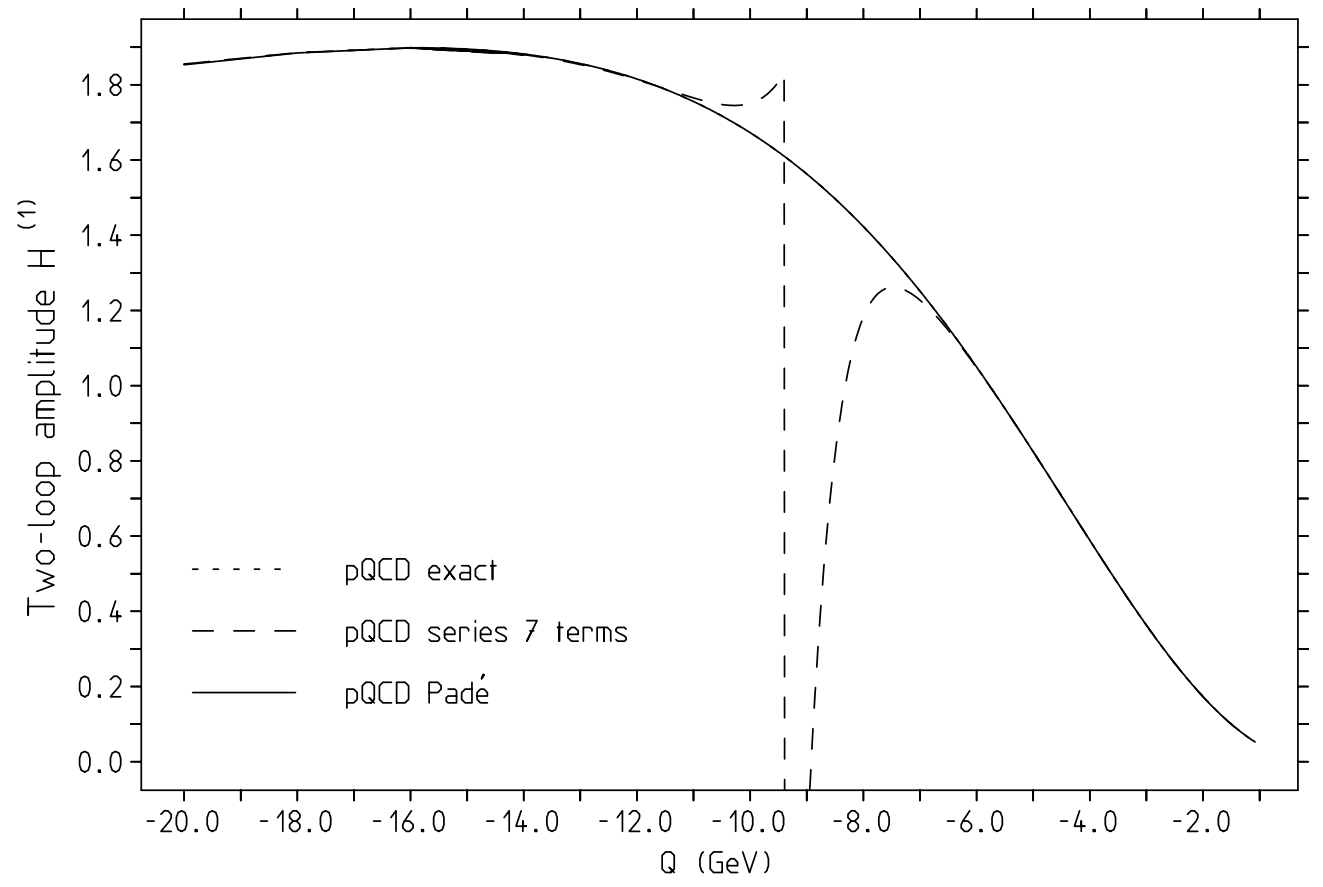

Figure 3: The two-loop amplitude $H(1)=\left(12 \pi^{2}\right) \dot{\Pi}_{V}^{\prime(2)}$ : shown is the exact result (dotted line), the series expansions for low $Q^{2}$ (8 terms) $\left(4 m^{2}>Q^{2}\right)$ and for large $Q^{2}$ (7 terms) $\left(Q^{2}>4 m^{2}\right)$ (dashed line) and the Padé improvement (full line) of the low $Q^{2}$ series working excellent up to $\left(16 m^{2}>Q^{2}\right)$. For higher $Q^{2}$ the large $Q^{2}$-expansion works perfectly. The dotted line is not seen because of the perfect agreement between the exact result and the Padé improved approximation. 
from the complex negative $q^{2}$ half-plane to the interior of the unit circle $|\omega|<1$ together with Padé resummation as proposed in [21]. The Padé approximant provides a good estimation to much higher values of $1 / y$ up to about $1 / y \sim 4$. This is displayed in Fig. 3, which shows that utilizing the Padé improvement allows us to obtain reliable results also in the relevant Euclidean "threshold region", around $y=1$.

The three-loop vector current amplitude $\Pi^{\prime(3)}$ is available in the form of a small $Q^{2}$ expansion, Eq. (47) of Ref. [3], and as a large $Q^{2}$ expansion, Eqs. (7-10) of Ref. [4]. By the definition (4) the contribution to the Adler function is obtained by differentiation

$$
\dot{\Pi}^{\prime(3)}(y)=-y d \Pi^{\prime(3)}(y) / d y
$$

While the low $Q^{2}$ expansion is given in the on-shell scheme, where it is a simple power series in $1 / y$, the high $Q^{2}$ expansion is given in the $\overline{\mathrm{MS}}$ scheme as an expansion in $\bar{y}=4 \bar{m}^{2} / Q^{2}$ where $\bar{m}=\bar{m}\left(\mu^{2}\right)$ is the $\overline{\mathrm{MS}}$ running mass at scale $\mu$ and the $\overline{\mathrm{MS}}$ amplitude $\bar{\Pi}^{\prime}(3)$ includes powers of the logarithms $l_{q m} \equiv \ln \left(-q^{2} / \bar{m}^{2}\right)$ and $l_{q \mu} \equiv \ln \left(-q^{2} / \mu^{2}\right)$ in additions to powers of $\bar{y}$.

Since, for simplicity, we work with pole masses, we have to reparametrize the $\overline{\mathrm{MS}}$ amplitude. The relationship between the $\overline{\mathrm{MS}}$ mass $\bar{m}$ and the pole mass $m$ is given by [3, 34, 35]

$$
m=\bar{m}\left(1+c_{1}\left(\frac{\alpha_{s}}{4 \pi}\right)+c_{2}\left(\frac{\alpha_{s}}{4 \pi}\right)^{2}+\ldots\right)
$$

with

$$
\begin{aligned}
c_{1} & =C_{F}(4+3 L), \\
c_{2} & =C_{F} C_{A}\left(\frac{1111}{24}-8 \zeta_{2}-4 I_{3}(1)+\frac{185}{6} L+\frac{11}{2} L^{2}\right) \\
& -C_{F} T_{F} n_{f}\left(\frac{71}{6}+8 \zeta_{2}+\frac{26}{3} L+2 L^{2}\right) \\
& +C_{F}^{2}\left(\frac{121}{8}+30 \zeta_{2}+8 I_{3}(1)+\frac{27}{2} L+\frac{9}{2} L^{2}\right)-12 C_{F} T_{F}\left(1-2 \zeta_{2}\right) .
\end{aligned}
$$

where $I_{3}(1)=\frac{3}{2} \zeta_{3}-6 \zeta_{2} \ln 2, L=\ln \left(\mu^{2} / m^{2}\right), C_{A}=3, C_{F}=4 / 3, T_{F}=1 / 2$ and $n_{f}=6$.

Asymptotically, for large $Q^{2}$ we find $\left(l_{q \mu} \equiv \ln \left(-q^{2} / \mu^{2}\right)\right)$

$$
\begin{aligned}
H^{(2)}(\infty) & =\frac{1}{32}\left\{\left(-3 C_{F}^{2}+\left(123-88 \zeta_{3}-22 l_{q \mu}\right) C_{A} C_{F}\right.\right. \\
& \left.\left.+\left(-44+32 \zeta_{3}+8 l_{q \mu}\right) C_{F} T_{F} n_{l}+\left(-44+32 \zeta_{3}+8 l_{q \mu}\right) C_{F} T_{F}\right)\right\}
\end{aligned}
$$

which coincides with the $\overline{\mathrm{MS}}$ expression for the $\alpha_{s}^{2}$ coefficient in the $D$-function, calculated in Ref.[13]. At the scale $\mu^{2}=Q^{2}$ is of order unity: $H^{(2)}(\infty) \simeq 1.40923$.

Due to the $\operatorname{logs} \ln \left(Q^{2} / m^{2}\right)$, still present after setting $\mu^{2}=Q^{2}$ in the three-loop amplitudes, we perform the Padé improvement for the non-log and the log terms separately. The result is depicted in Fig. 4. 
Note that the Padé approximant is an analytic continuation of the small $Q^{2}$ expansion with 8 terms. From a practical point of view it provides the "exact" mass dependence of the three-loop amplitude.

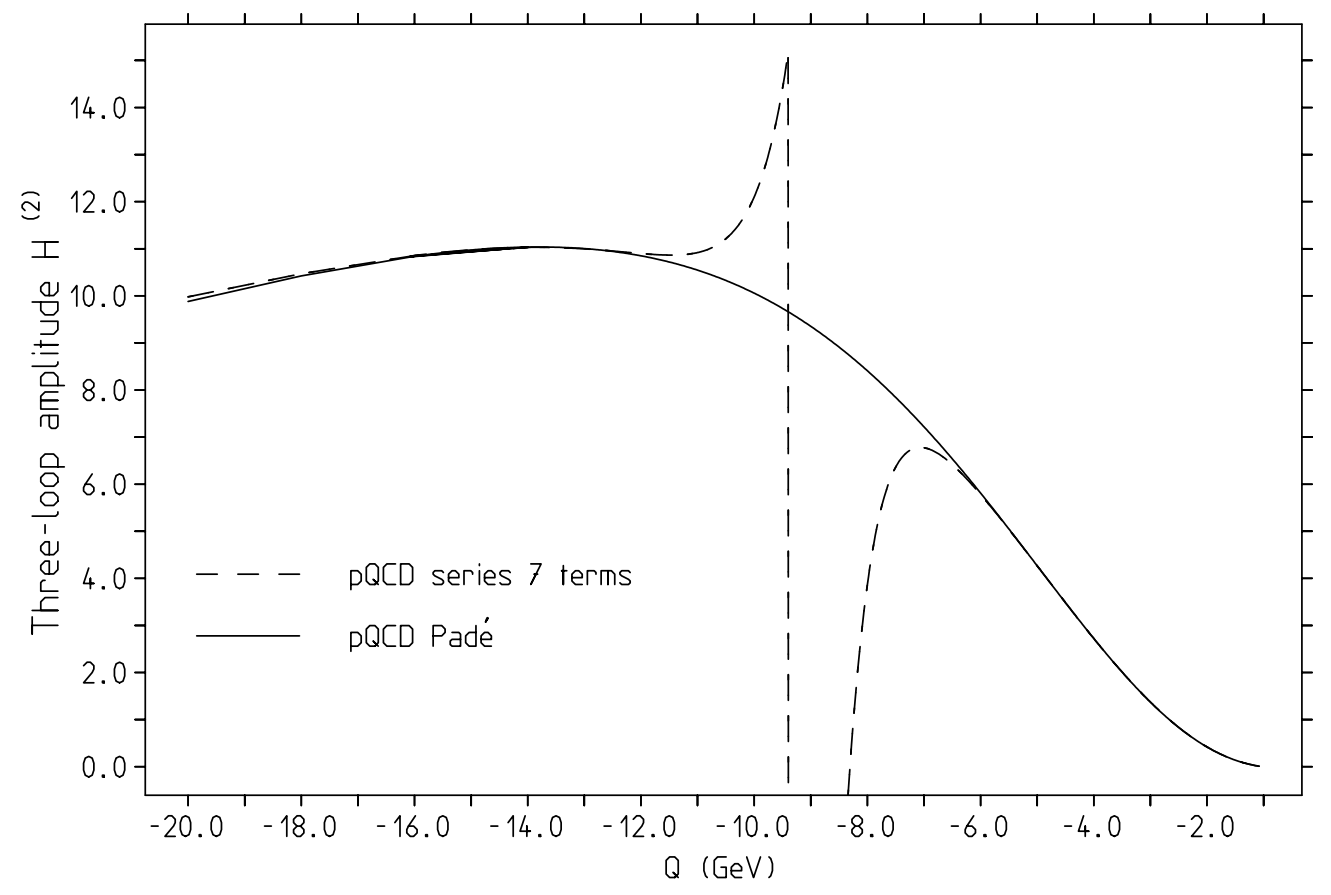

Figure 4: The three-loop amplitude $H^{(2)}=\left(12 \pi^{2}\right) \dot{\Pi}_{V}^{\prime(3)}$ : shown are the series expansions for low $Q^{2}$ (8 terms) $\left(4 m^{2}>Q^{2}\right)$ and for large $Q^{2}(7$ terms $)\left(Q^{2}>4 m^{2}\right)$ (dashed line) and the Padé improvement (full line) of the low $Q^{2}$ series working excellent up to $\left(16 \mathrm{~m}^{2}>Q^{2}\right)$. For higher $Q^{2}$ the large $Q^{2}$-expansion works perfectly.

\section{References}

[1] S.I. Eidelman and F. Jegerlehner, Z. Phys. C 67 (1995) 585;

F. Jegerlehner, Nucl. Phys. B (Proc. Suppl.) 51C (1996) 131.

[2] F. Jegerlehner and O.V. Tarasov, DESY 98-093 (hep-ph/9809485) and references therein.

[3] K.G. Chetyrkin, J.H. Kühn and M. Steinhauser, Nucl. Phys. B 482 (1996) 213.

[4] K.G. Chetyrkin, R. Harlander, J.H. Kühn and M. Steinhauser, Nucl. Phys. B 503 (1997) 339.

[5] H. Poggio, H. Quinn and S. Weinberg, Phys. Rev. D 13 (1976) 1958.

[6] R. Barnett, M. Dine and L. McLerran, Phys. Rev. D 22 (1980) 594.

[7] M.A. Shifman, A.I. Vainshtein and V.I. Zakharov, Nucl.Phys. B 147 (1979) 385.

[8] R. Bertelman, G. Launer and E. De Rafael, Nucl. Phys. B 250 (1985) 61.

[9] K.G. Chetyrkin, N.V. Krasnikov and A.N. Tavkhelidze, Phys. Lett. 76B (1978) 83. 
[10] R. Shankar, Phys. Rev. D 15 (1977) 755.

[11] S.I. Eidelman, L.M. Kurdadze and A.I. Vainshtein, Phys.Lett. 82B (1979) 278.

[12] C. Caso et al.(Particle Data Group), Eur. Phys. J. C 3 (1998) 1.

[13] K.G. Chetyrkin, A.L. Kataev and F.V. Tkachov, Phys. Lett. 85B (1979) 277;

M. Dine and J. Sapirstein, Phys. Rev. Lett. 43 (1979) 668;

W. Celmaster and R.J. Gonsalves, Phys. Rev. Lett. 44 (1980) 560.

[14] S.G. Gorishny, A.L. Kataev and S.A. Larin, Phys. Lett. B 259 (1991) 144;

L.R. Surguladze and M.A. Samuel, Phys. Rev. Lett. 66 (1991) 560; ibid. 2416 (Err), K.G. Chetyrkin, Phys. Lett. B 391 (1997) 402.

[15] S.G. Gorishny, A.L. Kataev and S.A. Larin, Nuov. Cim. 92A (1986) 119.

[16] S. Adler, Phys. Rev. D 10 (1973) 3714.

[17] A. De Rujula and H. Georgi, Phys. Rev. D 13 (1976) 1296.

[18] A.L. Kataev, Invited talk at the II Workshop "Continuous Advances in QCD", Minneapolis, March 1996, World Scientific 1996, ed. M.I.Polikarpov, p.107. (hep-ph/9607426).

[19] S. Peris, M. Perrottet and E. de Rafael, J. High Energy Phys. 05 (1998) 011.

[20] K.G. Chetyrkin and J.H. Kühn, Phys. Lett. B 342 (1995) 356.

[21] J. Fleischer and O.V. Tarasov, Z. Phys. C 64 (1994) 413.

[22] D.J. Broadhurst and S.C. Generalis, Open University preprint OUT-4102-12 (1984) (unpublished); S.C. Generalis, J. Phys. G 15 (1989) L225.

[23] K.G. Chetyrkin, S.G. Gorishny and V.P. Spiridonov, Phys. Lett. 160B (1985) 149.

[24] V.P. Spiridonov and K.G. Chetyrkin, Yad. Fiz. 47 (1988) 818 [Sov. J. Nucl. Phys. 47 (1988) 522]

[25] L.R. Surguladze and F.V. Tkachov, Nucl. Phys. B 331 (1990) 35.

[26] S. Narison, "QCD Spectral Sum Rules", (Lecture Notes in Physics, Vol. 26), World Scientific, Singapore 1989.

[27] H.G. Dosch and S. Narison, Phys. Lett. B 417 (1998) 173.

[28] J. Gasser, and H. Leutwyler, Phys. Rep. C 87 (1982) 77;

J. Bijnens, J. Padres and E. de Rafael, Phys. Lett. B 348 (1995) 226;

H. Leutwyler, Phys. Lett. B 378 (1996) 313.

[29] D. Karlen, Experimental Status of the Standard Model, talk presented at the "XXIX International Conference on High-Energy Physics" (ICHEP 98), Vancouver 1998.

[30] F. Jegerlehner, Prog. Part. Nucl. Phys. 71 (1991) 1, Sec.4.5. 
[31] W. Bernreuther and W. Wetzel, Nucl. Phys. B 197 (1983) 228; ibid. B 513 (1998) 758 (Err);

W.J. Marciano, Phys. Rev. D 29 (1984) 580;

S.A. Larin, T. van Ritbergen and J.A.M. Vermaseren, Nucl. Phys. B 438 (1995) 278;

K.G. Chetyrkin, B.A. Kniehl and M. Steinhauser, Phys. Rev. Lett. 74 (1997) 2184;

G. Rodrigo, A. Pich, and A. Santamaria, Phys. Lett. B 424 (1998) 367.

[32] J. Blümlein and W.L. van Neerven, DESY-98-176, hep-ph/9811351.

[33] T.H.Chang, K.J.F.Gaemers and W.L. van Neerven, Nucl. Phys. B 202 (1982) 407;

L.J. Reinders, H.R. Rubinstein and S. Yazaki, Phys. Rep. C 127 (1985) 1;

B.A. Kniehl, Nucl. Phys. B 347 (1990) 86.

[34] N. Gray, D.J. Broadhurst, W. Grafe and K. Schilcher, Z. Phys. C 48 (1990) 673.

[35] J. Fleischer, F. Jegerlehner, O.V. Tarasov and O.L. Veretin, DESY 98-026, hepph/9803493, Nucl. Phys. B 539 (1999) to appear. 